\title{
REVIEW
}

\section{Antimicrobial Stewardship in Australian Hospitals and Other Settings}

Kelly A. Cairns · Jason A. Roberts · Menino O. Cotta · Allen C. Cheng

To view enhanced content go to www.infectiousdiseases-open.com

Received: June 25, 2015 / Published online: September 11, 2015

(C) The Author(s) 2015. This article is published with open access at Springerlink.com

\section{ABSTRACT}

Australia's model of antimicrobial stewardship (AMS) has evolved significantly over recent years, from a long-standing national approach of antimicrobial prescribing guidelines and antimicrobial prescribing restrictions to recent advances including the first National Antimicrobial Resistance Strategy and incorporating mandatory AMS as part of hospital accreditation standards. AMS programs are most commonly found in the hospital setting. Various models are used throughout the country based on the local

K. A. Cairns $(\bowtie)$

Pharmacy Department, Alfred Health, Melbourne, VIC, Australia

e-mail: k.cairns@alfred.org.au

J. A. Roberts · M. O. Cotta

Department of Intensive Care Medicine and

Pharmacy Department, Royal Brisbane and

Women's Hospital, Brisbane, QLD, Australia

J. A. Roberts - M. O. Cotta

Burns Trauma and Critical Care Research Centre, The University of Queensland, Brisbane, QLD, Australia

J. A. Roberts · M. O. Cotta

School of Pharmacy, The University of Queensland, Brisbane, QLD, Australia context and resources available. Programs implemented at Alfred Health and the Royal Brisbane and Women's Hospital represent two successful models in tertiary referral settings that accommodate a general ward setting as well as specialized areas with a high infection burden. Measurement of outcomes related to AMS activities remains poorly standardized, with process indicators such as antimicrobial utilization forming a large proportion of outcome measurement. Presently there is no requirement for any AMS outcome measurements to be reported externally. Point prevalence surveys of appropriateness of

\section{O. Cotta}

Department of Medicine, Royal Melbourne

Hospital, University of Melbourne, Melbourne, VIC, Australia

A. C. Cheng

Infection Prevention and Healthcare Epidemiology Unit, Alfred Health, Melbourne, VIC, Australia

\section{A. C. Cheng}

Department of Epidemiology and Preventive Medicine, Monash University, Melbourne, VIC, Australia 
prescribing and compliance with prescribing guidelines are widely used at a national level. Despite this, there is still a paucity of published Australian data to support the effect of AMS on patient clinical outcomes. Private hospitals, the community, veterinary medicine and aged care sectors represent an important area for future AMS expansion within Australia. The AMS focus has traditionally been on prescribing restrictions (through the Commonwealth funding agencies); however, recent work has described other areas for improvement and development in both settings. AMS in Australia continues to evolve. The recent development of an Australian strategic plan to link antimicrobial utilization and resistance surveillance with policy represents an important step forward for the future of AMS in Australia.

Keywords: Antimicrobial stewardship; Australia; Infection prevention and control; Resistance

\section{INTRODUCTION}

Australia's model of antimicrobial stewardship (AMS) has evolved over time and has adapted to the organization and funding of the healthcare system, the coordination and availability of resources and prevalence of resistant microorganisms. Australia has had a long history of drug regulation and coordinated policy that has formed a solid foundation for AMS to be developed upon. Australia has responded to the global need for AMS and increased awareness, with significant advances made in the last 5 years.

This article is based on previously conducted studies and existing collections of nonidentifiable data and therefore is exempted from the need for ethical review according to the Australian National Statement on Ethical Conduct in Human Research $(5.1 .22,2007)$.

\section{THE AUSTRALIAN CONTEXT}

In Australia's healthcare system, there is a division between primary care (including outpatient pharmaceutical reimbursement), which is funded by the Federal government, and hospital care (including inpatient drug costs) primarily operated by State and Territory governments. In Australia, around $70 \%$ of healthcare is provided by government-funded public hospitals, with private hospitals providing significant services, mostly in obstetric and elective surgery [1].

Australia has had a long history of standardized national treatment guidelines, which allows for the implementation of policies nationally across all levels of healthcare, including primary care and hospitals. These are developed by Therapeutic Guidelines, an independent not-for-profit organization that engages clinicians to review and update the literature. National guidelines have been an effective tool when combined with broader regulatory policies [2]. The National Prescribing Service (NPS), a government-funded organization, has implemented quality use of medicines initiatives targeted at primary care professionals and consumers [3].

Australia also has a strong system of hospital accreditation standards analogous to the Joint Commission in the USA. Of the ten National Standards developed by the Australian Commission on Safety and Quality in Healthcare, one standard focuses on preventing and controlling healthcare-associated infections (standard 3) and from 2013 onwards has included 
substandard 3.14, which is specific to AMS [4]. A recently drafted set of Clinical Care Standards has been developed providing recommendations for the quality use of antimicrobials [5].

The Australian healthcare system also has a number of limitations that remain challenges for implementation of AMS. Australia is yet to widely implement an electronic medical record despite government interest in this area [6]. Although electronic medical records are widely used in primary care, a number of different commercial systems are used, which is a significant barrier to information exchange. Electronic medical records and prescribing systems are not yet commonplace in Australian hospitals.

Surveillance for antimicrobial use and antibiotic resistance is piecemeal and relies on a patchwork of small systems. The Australian Group on Antimicrobial Resistance (AGAR) has performed centralized testing for selected organisms, but this system is also voluntary and based primarily on data from large hospitals, which may bias reported results [7]. Public health surveillance systems cover resistance in bacterial notifiable diseases including pneumococci, tuberculosis, enteric pathogens and Neisseria spp.

However, a number of recent developments have provided a high-level policy framework to coordinate the current patchwork of surveillance and policy. After many years in abeyance following the pioneering work of the Joint Expert Technical Advisory Committee on Antibiotic Resistance (JETACAR) [8] and Expert Advisory Group on Antimicrobial Resistance (EAGAR) [2], a national government committee was established in 2012 to develop a national strategy for antimicrobial resistance following an Antimicrobial Resistance Summit in 2011 [9, 10]. This group has developed the first national strategy in June 2015 [11] highlighting education, coordinated surveillance and AMS across human and animal health, infection prevention and research as key to Australia's response.

\section{AMS IN HOSPITAL SETTINGS}

\section{The Development of Hospital AMS Models in Australia}

The Australian Commission on Safety and Quality in Healthcare first published guidelines for AMS in Australian Hospitals in 2011 [12]. These guidelines include the key components essential for an AMS program in Australia including antimicrobial formularies and approval systems, prescriber education and feedback, point of care interventions and measurement of outcomes. These guidelines, based on which many AMS programs are developed, are being revised at the time of writing.

Restriction policies have existed in many Australian hospitals for many years, but formal AMS programs, including governance structures and multidisciplinary teams, are a more recent development over the last 5-10 years. A survey of hospitals in 2008 found that $80 \%$ of 78 surveyed Australian hospitals had a restricted formulary for antimicrobial prescribing, but only $25 \%$ had a multidisciplinary AMS team [13]. By 2012, a Victorian survey found that formulary restriction, auditing and feedback to prescribers were implemented in a majority of public metropolitan hospitals, but were not in public regional hospitals or private hospitals [14]. A Queensland survey also found that formulary restrictions were the mainstay of AMS programs, with auditing and feedback to prescribers present in $76 \%$ of hospitals [15]. The 
incorporation of AMS into the National Accreditation Standards for Australian Hospitals in 2013 has mandated the need for AMS and has been a driving factor for the implementation and expansion of AMS in Australia.

\section{Models of AMS in Public Hospital Settings}

The program implemented at Alfred Health in Melbourne provides one example of a model of AMS. At this health service, which includes around 1000 hospital beds across three campuses, an AMS Committee includes representatives of pharmacy, infectious diseases, microbiology as well as key prescriber groups such as respiratory medicine, emergency medicine, intensive care and surgery. This committee oversees policies relating to antimicrobial use and resistance, including the development of local prescribing guidelines, and monitoring and evaluation of the program.

Elements of the AMS program include formulary restriction (requiring web-based approval for most broad-spectrum antimicrobials or specific approval from an infectious diseases physician for selected agents), education of prescribers and drug use evaluations and reporting to unit heads on trends in antimicrobial use. During the formative phase, work was undertaken to achieve consensus on approved indications for the use of antimicrobial agents within the hospital, review of the formulary restrictions and the availability of antimicrobial agents within ward areas of the hospital and to implement a web-based antimicrobial approval system. An AMS team was formed and includes a full time pharmacist as well as part time physicians with expertise in infectious diseases, clinical microbiology and epidemiology.

The AMS program has a different model of engagement with areas with a moderate burden of infection and antimicrobial use (including general medicine, most surgical units) and those with a high burden of infection and antimicrobial use (such as intensive care, haematology/bone marrow transplantation, burns and cystic fibrosis/lung transplantation). In the latter, the complex nature of patients and need for timely access to broad-spectrum antibiotics mitigates the effectiveness of pre-prescription models of AMS, such as formulary restriction.

In response to these limitations, AMS ward rounds performing prospective auditing with intervention and feedback for those units with a moderate burden of infection form a key focus of this program. This post-prescription model of AMS has resulted in a reduction in the volume of prescribing of several key classes of antimicrobial agents [16].

\section{Specialized Public Hospital Areas with a High Infection Burden (e.g., Intensive Care Units)}

The model of rapid audit and review is best suited to patients that have a moderate burden of infection and are of low to moderate complexity. A different model of AMS is required for highly complex patients with a high burden of infection, such as in intensive care, hematology/bone marrow transplant or cystic fibrosis/lung transplantation. At some Australian hospitals, infectious disease physicians are "embedded" in these units, performing conjoint ward rounds, liaising with microbiology and clinical consultation, as well as developing policies and clinical guidelines covering both treatment and prevention of infections in these groups.

An example of one model of AMS is that found in the intensive care unit at The Royal Brisbane and Women's Hospital. This hospital 
has 30 intensive care beds and over 900 hospital beds. The AMS activity is led by the intensive care consultants with at least daily interaction with nursing, pharmacy, infectious diseases and microbiology teams. Although the intensive care unit is a "closed" unit (intensive care clinicians only have prescribing, admission and discharge rights), it has strong relationships with other relevant disciplines. Twice weekly teaching management rounds occur with infectious diseases, microbiology and pharmacy team members reviewing all patients with new or persisting microbiological issues with attendance by infection control practitioners. Patient consults by infectious diseases physicians can occur up to daily as needed.

Antimicrobial susceptibility patterns are prepared annually by the Microbiology Department. Optimized dosing is facilitated by the Pharmacy Department in conjunction with the Chemical Pathology Department, which provides an extended therapeutic drug monitoring service (aminoglycosides, glycopeptides, beta-lactams, quinolones and triazole antifungals). Dose adjustment is supported by Bayesian dose optimization methods in particularly complex patients.

Supporting the daily interactions of the relevant team members is a strong program of research, audit and guideline development led by the intensive care team (medical, nursing and pharmacy staff). Involvement of relevant specialties is also an important component of this work.

\section{MONITORING AND EVALUATION OF HOSPITAL AMS PROGRAMS}

Assessment and measurement of the impact of AMS clinical services in Australia remains poorly standardized. Indicators used in Australian hospitals include process indicators, volume-based measures of antimicrobial use and quality-based indicators of antimicrobial use. Recently, clinical care standards for AMS were released by the Australian Commission on Safety and Quality in Healthcare, providing useful guidance to hospitals to monitor and evaluate AMS programs [5].

\section{Process Indicators}

In-house, site-specific data are often kept by AMS teams, and currently there is no requirement to report this externally. While cost has been used to justify AMS programs, the marked reduction in purchasing costs of broad-spectrum antimicrobial agents within Australia as generic formulations have become available, suggests that this approach is unlikely to be as successful and that other process outcomes should be used for evaluation. Some processes monitored at Alfred Health include use of the electronic approval/alert system (see Fig. 1a), the number of patients reviewed by an AMS team per month and the number of recommendations made by an AMS team per month (see Fig. 1b). In addition, data are reviewed on hospital-wide antimicrobial utilization and changes in broad-spectrum antimicrobial use [16].

\section{Antimicrobial Use}

Many hospitals contribute data on a voluntary basis on antimicrobial utilization to the National Antimicrobial Utilisation Surveillance Program (NAUSP). These data are based on pharmacy dispensing and distribution data using the World Health Organization (WHO)-defined daily dose method [17]. Although they provide useful data on 

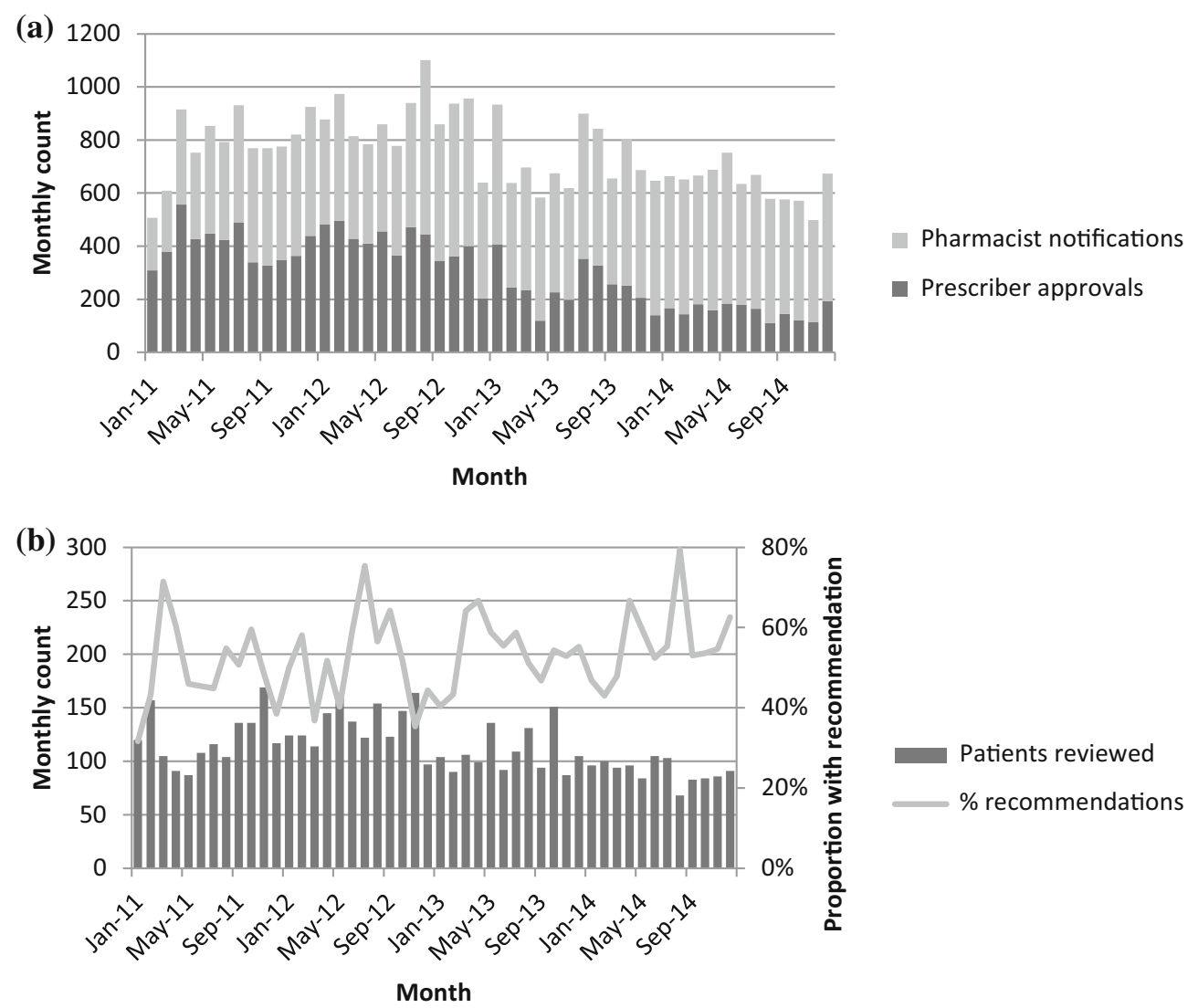

Fig. 1 Selected antimicrobial stewardship program process indicators at Alfred Health. a Monthly number of prescriber electronic approvals and pharmacist notifications for use of restricted antimicrobials.

prescribing trends over time, the nature of the data source results in considerable month-to-month variation, reflects the total volume rather than the number of prescriptions and does not provide data on the appropriateness of use. In particular, benchmarking between hospitals is difficult because of variation in the patient case mix.

\section{Point Prevalence Surveys}

The National Antimicrobial Prescribing Survey (NAPS) is an annual point prevalence survey undertaken in November of each year to b Monthly number of patients reviewed by audit/feedback rounds (left axis) and proportion of patients seen where a recommendation was made to change antimicrobial therapy (right axis)

coincide with Antimicrobial Awareness Week [18]. This survey includes items to assess the appropriateness of antimicrobial prescription and compliance with guidelines (either national or local). It has been conducted by an increasing number of hospitals on a voluntary basis since 2011 [18]. In 2013, it identified this at 151 contributing hospitals (encompassing approximately 12,800 individual antimicrobial prescriptions). At these hospitals, $70.8 \%$ of antimicrobial prescriptions were considered to be appropriately prescribed, while $59.7 \%$ of prescriptions were classed as compliant with national or endorsed local guidelines [19]. 


\section{Clinical Outcomes}

There has been a paucity of published AMS studies focusing on clinical outcomes in the Australian setting to date. The generalizability of clinical studies from international publications is limited because of differences in infrastructure, resource availability and antimicrobial resistance profiles. While the link between antimicrobial use and resistance is unambiguous mechanistically and on an ecological scale, it has been more difficult to demonstrate this link in hospital settings, probably because of the many potential confounders to this association. In Australia, some studies have found a strong correlation between decreasing antibiotic use and decreasing gram-negative resistance following a successful AMS program [20], but other studies have failed to find associations between broad-spectrum antimicrobial utilization following the introduction of an AMS program and observed rates of Clostridium difficile infection [21].

\section{Strengths and Limitations of Public Hospital AMS Programs}

Both our institutions considered the use of pre-prescription and point of prescription interventions. However, a program that relies largely on antimicrobial restriction may experience conflict with the need for timely antimicrobial therapy, particularly in patients with severe sepsis. Embedding authorization with decision support tools has been implemented, but in the absence of electronic prescribing, it is not sustainable as it is not embedded in prescriber workflow.

A key strength of programs that rely on post-prescription audit and feedback includes the recognition that the individual patient clinical situation and subsequent decision-making process are complex. Not all patients can be encapsulated within clinical guidelines, with significant patient-specific factors requiring consideration. High visibility and regular post-prescription antimicrobial review enable insight into many aspects of antimicrobial prescribing and use that may not be recognized through other more passive mechanisms of review, including prophylaxis regimens and noninfectious use of antimicrobial agents. Regular AMS rounds provide regular teaching moments to junior medical and pharmacy staff and enable increased awareness and education about infectious diseases and AMS principles.

In our experience, some limitations of the current AMS programs include the inability at present to link the AMS activities with direct changes in both local and national antimicrobial utilization, changes in antimicrobial resistance rates and improvements in $C$. difficile rates. This is exacerbated by the current piecemeal nature of national antimicrobial surveillance. Capturing of the exact antimicrobial utilization rates is also limited by the absence of electronic prescribing so that use can only be tracked to a ward rather than the individual patient level. An ongoing challenge for all AMS programs is maintaining momentum and ensuring the sustainability of the program. Finally, the labor-intensive nature of this program is a major limitation to further development of the program.

\section{AMS IN OTHER SETTINGS}

\section{Private Hospitals}

Although around $30 \%$ of all inpatient hospital care in Australia occurs in the private hospital sector [1], AMS in private hospitals has lagged 
behind that seen in public hospitals [14]. There are significant cultural and organizational differences for private hospitals when compared to the Australian public sector, which holds important implications for the model of AMS that is feasible in this setting. Principally, private hospitals often view their role as facilitating and servicing medical specialists' practices. This is reflected in the relatively autonomous practices of specialists, the role of pharmacists in simply providing medication rather than taking an active role in medication management and the relative lack of hospital-wide policies and coordinators of AMS activities [22]. For example, infectious diseases physicians are typically solely responsible for their own patients, with no additional hospital-wide role such as in infection control or AMS. Likewise, clinical microbiologists (employed by outsourced pathology services) and pharmacists may be limited to a supply function and do not have jurisdiction to oversee AMS programs in private hospitals [22].

There is, however, significant opportunity for the private sector to tailor potential solutions for successful AMS program implementation. Establishing an antimicrobial prescribing policy that makes specialists aware of their responsibilities for judicious antimicrobial use by ensuring the prescribing policy forms part of their renewal for accreditation to admit patients can send a powerful message from hospital executive. Pharmacists have been shown to be important advocates of AMS [23], and there is significant scope for them to lead post-prescription review and periodic auditing if given the mandate to do so. Due to their pivotal role in private hospitals, nursing staff will clearly need to be part of any AMS program in private hospitals but further work is required to provide both the awareness, training and authority to effect change [22].

\section{Primary Care}

\section{Pharmaceutical Benefits Scheme}

Australia has a public subsidy for drugs through the national Pharmaceutical Benefits Scheme (PBS), and this has an important influence on antimicrobial prescribing in Australia. The effect of the PBS has best been demonstrated for fluoroquinolones, where a coordinated national policy has restricted indications for use in national treatment guidelines, the requirement for an 'authority' to be obtained to prescribe fluoroquinolones on the PBS (medical practitioners need to call a prescribing approval service to obtain approval to prescribe) and the banning of fluoroquinolone use in food-producing animals [2]. This policy has resulted in a low rate of fluoroquinolone use in Australia and a relatively low rate of resistance to this valuable class of antimicrobials [2].

The Drug Utilisation Subcommittee of the Pharmaceutical Benefits Advisory Committee recently released a review of the use of antibiotics in primary care in Australia [24]. In 2013, they reported that $45 \%$ of the Australian population were supplied at least one antibiotic and that the overall utilization of antimicrobials in Australia (22.8 Defined Daily Doses (DDDs)/1000 person days) was higher than the Organisation for Economic Cooperation and Development average (21.1 DDDs/1000 person days) [24]. The three most commonly prescribed antimicrobial agents were amoxycillin, cephalexin and amoxycillin/clavulanate combinations [24]. 


\section{National Prescribing Service}

The NPS is an independent, government-funded organization founded in 1998 to improve the quality use of medicines as a component of the National Medicines Policy [25]. It aims to change attitudes and behaviors that exist around the use of medicines, and initiatives have included antimicrobials in a current campaign that commenced in 2012. They provide educational programs to around half of Australia's general practitioners through initiatives such as academic detailing and clinical self-audits of prescribing practice. The NPS is also providing broad consumer education about antimicrobial resistance in a current "Resistance Fighter" campaign [3].

\section{Aged Care}

In contrast to other countries, there are few acute/subacute care long-term care facilities in Australia, but around $8.5 \%$ of the elderly $>70$ years live in residential aged care facilities (RACFs), with various levels of nursing support [26]. With increasing awareness of AMS in recent years, the importance of RACFs as a reservoir for multidrug-resistant (MDR) organisms is becoming more apparent. A 2011 study by Stuart et al. identified low rates of vancomycin-resistant enterococci (VRE) and $C$. difficile colonization in a cohort of 119 patients in Victoria, but a high prevalence of extended-spectrum beta-lactamase (ESBL)-producing Escherichia coli [27]. Similarly, another Melbourne study found that $36 \%$ of RACF residents were colonized with methicillin-resistant Staphylococcus aureus (MRSA), VRE or MDR gram-negative bacilli [28].

There is no ongoing surveillance of antimicrobial use in Australian RACFs, but one 4-year Melbourne study has shown that the majority of antimicrobial agents used were oral, narrow-spectrum agents such as cephalexin and trimethoprim [29]. However, it also highlighted that a significant proportion of prescribing appeared to occur in patients without obvious, documented clinical findings suggestive of infection and that none of the participating RACFs had antimicrobial restriction policies in place. Qualitative work has identified a number of barriers and enablers to AMS in this setting [30]. Workflow-related factors included the lack of on-site medical care, pharmacy support, nurse-driven infection management, institutional policies and guidelines, and external expertise and diagnostic facilities. A subsequent survey found that stakeholders were generally supportive of AMS interventions and potential strategies included nursing-based education, aged-care-specific antibiotic guidelines and regular antibiotic surveillance [31].

\section{Veterinary Medicine}

The role of AMS in veterinary medicine remains in its infancy in Australia and lags behind that of the human sector. There are currently no nationally coordinated veterinary or agricultural antimicrobial resistance monitoring and surveillance programs in Australia; however, a range of activities is underway to address this issue, including the Australian One Health Antimicrobial Resistance Colloquium [32]. Historical sales data are available between 2005 and 2010, with a staggering 500-600 tons of active antimicrobial agents being sold on average per annum for the animal sector, with $98 \%$ of these antimicrobials being used in food-producing animals [33]. Approximately $40 \%$ of antimicrobials were used for therapeutic effect in animals, while $4-7 \%$ were used for growth 
promotion. There have been attempts to restrict the veterinary use of antibiotics deemed to be important therapeutic agents in human medicine; significantly, fluoroquinolones are not approved for use in Australian food animals $[2,34]$.

\section{CONCLUSIONS}

AMS programs in Australia have evolved from informal restriction policies to more comprehensive programs with a formal governance structure and multidisciplinary teams. More broadly, an Australian strategic plan has recently been developed to link surveillance with policy across the various domains of the healthcare system including both antimicrobial use and antimicrobial resistance, from primary care to aged care to hospitals. The strategy also recognizes that surveillance and AMS are required in animal health. While some elements are similar, different models of engagement are required in different settings. Some examples of successful strategies include regulation and treatment guidelines in primary care, nursing-led infection management in aged care, and post-prescription audit and feedback in hospitals. Further work is required to refine and evaluate these and other models of AMS in Australia.

\section{ACKNOWLEDGMENTS}

This supplement was not sponsored by outside commercial interests. Jason Roberts is funded, in part, by a National Health and Medical Research Council Research Fellowship (APP1048652). Allen Cheng is also supported by a National Health and Medical Research Council Career Development Fellowship. No source of funding was received for publication of this article. All named authors meet the International Committee of Medical Journal Editors (ICMJE) criteria for authorship for this manuscript, take responsibility for the integrity of the work as a whole and have given final approval for the version to be published.

Conflict of interest. Kelly A. Cairns, Jason A. Roberts, Menino O. Cotta and Allen C. Cheng have nothing to disclose.

Compliance with ethics guidelines. This article is based on previously conducted studies and existing collections of non-identifiable data and therefore is exempted from the need for ethical review according to the Australian National Statement on Ethical Conduct in Human Research $(5.1 .22,2007)$.

Open Access. This article is distributed under the terms of the Creative Commons Attribution-NonCommercial 4.0 International License (http://creativecommons.org/licenses/ by-nc/4.0/), which permits any noncommercial use, distribution, and reproduction in any medium, provided you give appropriate credit to the original author(s) and the source, provide a link to the Creative Commons license, and indicate if changes were made.

\section{REFERENCES}

1. Australian Institute of Health and Welfare (AIHW). Australia's health system [Internet]. Canberra: AIHW; 2014 [cited 13 June 2015]. http://www. aihw.gov.au/australias-health/2014/health-system/.

2. Cheng AC, Turnidge J, Collignon P, et al. Control of fluoroquinolone resistance through successful regulation, Australia. Emerg Infect Dis. 2012;18(9):1453-60. 
3. National Prescribing Service. Join the fight against antibiotic resistance [Internet]. Sydney NSW: NPS MedicineWise; 2014 [updated 20 Nov 2014; cited 13 June 2015]. http://www.nps.org.au/medicines/ infections-and-infestations/antibiotics/for-individuals/ join-the-fight-against-antibiotic-resistance.

4. Australian Commission on Safety and Quality in Health Care (ACSQHC). National Safety and Quality Health Service Standards [Internet]. Sydney (AU): ACSQHC; 2012 [cited 13 June 2015]. 76 p. http://www.safetyandquality.gov.au/wpcontent/uploads/2011/09/NSQHS-Standards-Sept2012.pdf.

5. Australian Commission on Safety and Quality in Health Care (ACSQHC). Antimicrobial Stewardship Clinical Care Standard [Internet]. Sydney (AU): ACSQHC; 2014 [cited 13 June 2015]. 19 p. http:// www.safetyandquality.gov.au/our-work/clinicalcare-standards/antimicrobial-stewardship-clinicalcare-standard/.

6. Victorian Department of Human Services. National E-Health Strategy [Internet]. Melbourne (AU): Victorian Department of Human Services; 2008 [cited 21 June 2015]. 31 p. http://health.gov.au/ internet/main/publishing.nsf/Content/69B9E0174 7B836DCCA257BF0001DC5CC/\$File/Summary\% 20National\%20E-Health\%20Strategy\%20final.pdf.

7. Australian Society for Antimicrobials. Australian Group for Antimicrobial Resistance (AGAR) Surveillance Programmes [Internet]. South Perth; 2015 [cited 13 June 2015]. http://www.asainc.net. au/aasp/agar.

8. Australian Department of Health. The use of antibiotics in food-producing animals: antibiotic-resistant bacteria in animals and humans [Internet]. Canberra ACT: Commonwealth of Australia; 2014 [updated 8 Dec 2014; cited 19 June 2015]. http://www.health.gov. $\mathrm{au} /$ internet/main/publishing.nsf/Content/healthpubs-jetacar-cnt.htm.

9. Gottlieb T, Nimmo GR. Antibiotic resistance is an emerging threat to public health: an urgent call to action at the Antimicrobial Resistance Summit 2011. Med J Aust. 2011;194(6):281-3.

10. Australian Department of Health. Antimicrobial resistance (AMR) [Internet]. Canberra ACT: Commonwealth of Australia; 2015 [updated 1 June 2015; cited 13 June 2015]. http://www. health.gov.au/internet/main/publishing.nsf/Content/ ohp-amr.htm.

11. Australian Government. Responding to the threat of antimicrobial resistance: Australia's First National Antimicrobial Resistance Strategy 2015-2019 [Internet]. Canberra (AU);
Commonwealth of Australia; 2015 [cited 13 June 2015]. 37 p. http://www.health.gov.au/internet/ main/publishing.nsf/Content/1803C433C71415CA CA257C8400121B1F/\$File/amr-strategy-2015-2019. pdf.

12. Duguid M, Cruickshank M. Antimicrobial stewardship in Australian hospitals. Sydney: Australian Commission on Safety and Quality in Health Care; 2010 [cited 13 June 2015]. 239 p. http://www.safetyandquality.gov.au/wp-content/ uploads/2011/01/Antimicrobial-stewardship-inAustralian-Hospitals-2011.pdf.

13. Chen AW, Khumra S, Eaton V, et al. Snapshot of antimicrobial stewardship in Australian hospitals. J Pharm Pract Res. 2010;40:19-25.

14. James RS, McIntosh KA, Luu SB, et al. Antimicrobial stewardship in Victorian hospitals: a statewide survey to identify current gaps. Med J Aust. 2013;199(10):692-5.

15. Avent ML, Hall L, Davis L, et al. Antimicrobial stewardship activities: a survey of Queensland hospitals. Aust Health Rev. 2014;38(5):557-63.

16. Cairns KA, Jenney AW, Abbott IJ, et al. Prescribing trends before and after implementation of an antimicrobial stewardship program. Med J Aust. 2013;198(5):262-6.

17. McNeil V, Cruickshank M, Duguid M. Safer use of antimicrobials in hospitals: the value of antimicrobial usage data. Med J Aust. 2010;193(8 Suppl):S114-7.

18. James R, Upjohn L, Cotta M, et al. Measuring antimicrobial prescribing quality in Australian hospitals: development and evaluation of a national antimicrobial prescribing survey tool. J Antimicrob Chemother. 2015;70(6):1912-8.

19. Australian Commission on Safety and Quality in Health Care. Antimicrobial Prescribing Practice in Australia: results of the 2013 National Antimicrobial Prescribing Survey [Internet]. Sydney (AU): ACSQHC; 2014 [cited 13 June 2015]. 25 p. http://www.safetyandquality.gov.au/wpcontent/uploads/2014/11/Web-Accessible-2013NAPS-Report.pdf.

20. Yong MK, Buising KL, Cheng AC, et al. Improved susceptibility of Gram-negative bacteria in an intensive care unit following implementation of a computerized antibiotic decision support system. J Antimicrob Chemother. 2010;65(5): 1062-9.

21. Cheng AC, Cairns KA, Jenney AW. Does antimicrobial stewardship impact on rates of Clostridium difficile? Paper presented at: The 
Scientific Meeting of the Australasian Society for Infectious Diseases, 26-29 Mar 2014, Adelaide, AU.

22. Cotta, MO, Robertson, MS, Marshall, C, et al. Implementing antimicrobial stewardship in the Australian private hospital system: a qualitative study. Aust Health Rev. 2015;39(3)315-322.

23. Cotta MO, Robertson MS, Tacey M, et al. Attitudes towards antimicrobial stewardship: results from a large private hospital in Australia. Healthc Infect. 2014;19(3):89-94.

24. Australian Department of Health. Drug Utilisation Sub Committee (DUSC) [Internet]. Canberra; 2014 [updated 24 Nov 2014; cited 13 June 2015]. http:// www.pbs.gov.au/info/industry/listing/participants/ drug-utilisation-subcommittee.

25. Australian Government: Department of Health and Aging. National Medicines Policy [Internet]. Canberra (AU): Commonwealth of Australia; 2000 [cited 13 June 2015]. 10 p. http://www.health.gov. au/internet/main/publishing.nsf/Content/B2FFBF7 2029EEAC8CA257BF0001BAF3F/\$File/NMP2000.pdf.

26. Australian Institute of Health and Welfare. Residential aged care in Australia 2010-2011: a statistical overview [Internet]. Canberra (AU): AIHW; 2012 [cited 13 June 2015]. 85 p. http:// www.aihw.gov.au/WorkArea/DownloadAsset.aspx? id $=10737422896$.

27. Stuart RL, Kotsanas D, Webb B, et al. Prevalence of antimicrobial-resistant organisms in residential aged care facilities. Med J Aust. 2011;195(9):530-3.

28. Lim CJ, Cheng AC, Kennon J, et al. Prevalence of multidrug-resistant organisms and risk factors for carriage in long-term care facilities: a nested case-control study. J Antimicrob Chemother. 2014;69(7):1972-80.

29. Lim CJ, McLellan SC, Cheng AC, et al. Surveillance of infection burden in residential aged care facilities. Med J Aust. 2012;196(5):327-31.

30. Lim CJ, Kwong MW, Stuart RL, et al. Antibiotic prescribing practice in residential aged care facilities-health care providers' perspectives. Med J Aust. 2014;201(2):98-102.

31. Lim CJ, Kwong M, Stuart RL, et al. Antimicrobial stewardship in residential aged care facilities: need and readiness assessment. BMC Infect Dis. $2014 ; 14: 410$.

32. Australian Commission on Safety and Quality in Health Care. Antimicrobial resistance: a report of the Australian one health antimicrobial resistance colloquium [Internet]. Sydney (AU): ACSQHC; 2013 [updated 2013; cited 21 July 2015]; 16 p. https://www.health.gov.au/internet/main/publishing. nsf/Content/1803C433C71415CACA257C8400121B1 F/\$File/Colloquium-Report-Final-Feb2014.pdf.

33. Australian Government: Australian Pesticides and Veterinary Medicines Authority (APVMA). Quantity of antimicrobial products sold for veterinary use in Australia [Internet]. Kingston (AU): APVMA; 2014 [updated 2014; cited 21 July 2015]. p. 81. http:// archive.apvma.gov.au/publications/reports/docs/ antimicrobial_sales_report_march-2014.pdf.

34. Australian Government: Australian Pesticides and Veterinary Medicines Authority (APVMA). Antibiotic resistance risk assessments [Internet]. APVMA; 2014 [updated 1 July 2014; cited 21 July 2015]. http://apvma.gov.au/node/1018. 